\title{
Antifertility effects of sperm impairing factors isolated from bacteria in male mice
}

\begin{abstract}
Sperm immobilizing (SIF) and sperm agglutinating factor (SAF) (already isolated in our laboratory) from Staphylococcus aureus and Escherichia coli, respectively, have shown 100 $\%$ infertility in female mice. These microbial factors neither induce any abnormalities in the vaginal cell morphology nor do they affect structural integrity of vaginal epithelium. Therefore, the present study was carried out to check any adverse effect of these factors on the reproductive potential of male mice. Mice were administered with SIF or SAF $(10 \mu \mathrm{g} / 20 \mu \mathrm{l})$ intraperitoneally for a period of 7 days on alternate days. No treatment related mortality was observed during the experiment. The mice were sacrificed $48 \mathrm{~h}$ post inoculation on days 3, 5 and 9 after intraperitoneal administration and the results in terms of change in body weight, seminal parameters, TSI and histology were studied. The results showed that male mice receiving SIF and SAF showed significant decrease in seminal parameters viz. sperm count, motility and viability w.r.t control mice. Histological studies of male mice revealed hypospermatogenesis and inflammation in the reproductive organs of treated mice while non reproductive organs showed normal histology. In conclusion, SIF/SAF upon intraperitoneal administration can lead to a pronounced decrease in male reproductive potential
\end{abstract}

Keywords: SIF, SAF, intraperitoneal, seminal parameters, histopathology
Volume 5 Issue 2 - 2017

Tanya Sharma, Aditi Chauhan, Deepali Thaper, Kalpana Rana, Sonia Gupta,Vijay Prabha

Department of Microbiology, Panjab University, India

Correspondence: Vijay Prabha, Professor Department of Microbiology, Panjab University, Chandigarh-1600 I4, India ,Tel 9|-I72-2534|40, Fax 91-172-2541770,

Email atishvijayII@yahoo.com

Received: May 18,2017 | Published: June 15, 2017

\section{Introduction}

Spermicides are known from centuries and the first written record of spermicide use is found in an Egyptian document namely the Kahun Papyrus, dating to 1850 BCE. ${ }^{1}$ Spermicide is a substance that kills or immobilizes the sperm and is inserted deep in the vagina near the cervix prior to intercourse to prevent pregnancy. Spermicides come in several forms such as gel, foam, cream, film, suppository or tablet. Most widely used spermicide is Nonoxynol-9 while other includes benzalkonium chloride, chlorhexidine, menfegol, octoxynol-9, and sodium docusate. The spermicidal activities of these detergent-type contraceptives are associated with their structural affinity to the membrane lipids ${ }^{2,3}$ therefore, the major drawback of using N-9 or other currently used surfactants is their detergent-type effect leading to an increased risk of vaginal or cervical infection, irritation or ulceration. In addition to this, alteration in the vaginal flora leads to an increased risk of opportunistic infections. ${ }^{4-6}$ In a systematic search for developing a new spermicide and microbicide, an earlier study on a microbial origin antimicrobial peptide nisin has shown good contraceptive efficacy in vitro as well as in vivo. ${ }^{7}$ The reproductive toxicity of nisin was also evaluated on the male rats where it was administered orally. ${ }^{8}$ On the other hand, Qin et al. ${ }^{9}$ demonstrated that when mice were given busulfan intraperitoneally, it resulted in deleterious effects on reproductive potential of male mice.

This intrigued us to evaluate the effect of microbial factors earlier isolated in our laboratory from Staphylococcus aureus and Escherichia coli, on the male mice which have shown excellent sperm impairment in vitro and caused $100 \%$ infertility in vivo in female mice. ${ }^{10,11}$ Thus the present study was designed to evaluate the effect of these microbial-derived biologically active spermicidal proteins on the reproductive potential of male mice.

\section{Materials and methods}

\section{Microorganisms}

The strains of both Staphylococcus aureus and Escherichia coli, isolated from the cervix of a women suffering from unexplained infertility and semen samples of infertile males undergoing semen analysis, respectively, were already available in the laboratory. The isolate of $S$. aureus showed sperm immobilization whereas $E$. coli showed sperm immobilization via agglutination in vitro.

\section{Experimental animals}

Sexually mature, 5-6 week old male $(25 \pm 2 \mathrm{~g}) \mathrm{BALB} / \mathrm{c}$ mice were used in the present study. The mice were maintained under standard laboratory conditions and were housed in propylene cages $\left(430 \times 270 \times 150 \mathrm{~mm}^{3}\right.$, six animals per cage $)$ at $20-25^{\circ} \mathrm{C}$, bedded with clean rice husk in well aerated animal room of the Department of Microbiology, Panjab University. All the animals were given standard pellet diet and water ad libitum. Animals were allowed to acclimatize to the new housing and experimental conditions for at least one week. All the experimental protocols were approved by the Institutional Animals Ethics Committee of the Panjab University Chandigarh vide letter no PU/IAEC/S/15/67 dated 15.09.2015 and were performed in accordance with the guidelines of committee for the purpose of Control \& Supervision of Experiments on Animals (CPCSEA), Government of India, on animal experimentation.

\section{Isolation and purification of sperm immobilization factor (SIF) from S. aureus}

SIF was extracted and purified from $72 \mathrm{~h}$ old cell culture of $S$. aureus by the method previously standardized in the laboratory. ${ }^{12}$ Briefly, the cell culture of $S$. aureus grown in brain heart infusion (BHI) broth, under shake conditions $(220 \mathrm{rpm})$ at $37^{\circ} \mathrm{C}$ for $72 \mathrm{~h}$, was centrifuged at $10,000 \mathrm{rpm}$ for $15 \mathrm{~min}$ at $4^{\circ} \mathrm{C}$. SIF was purified from the supernatant by ammonium sulphate precipitation, gel permeation chromatography, and ion exchange chromatography.

\section{Isolation and purification of Sperm agglutinating factor (SAF) from $E$. coli}

The SAF was extracted and purified from $48 \mathrm{~h}$ old cell culture of $E$. coli by the method earlier standardized in the laboratory. ${ }^{13}$ Briefly, the cell culture was washed with Phosphate buffered saline (PBS; $\mathrm{pH}$ 
$7.2,50 \mathrm{mM}$ ) and pellet was given salt treatment with $3 \mathrm{M} \mathrm{NaCl}$ for $12 \mathrm{~h}$ which released the factor into the solution. After dialysis and concentration of salt treated sample, further purification of SAF was carried out using gel filteration chromatography and ion exchange chromatography.

\section{Impact of intraperitoneal administration on reproductive potential of male mice}

Mice were divided into 3 groups with 9 mice in each group. Group 1, administered intraperitoneally with $20 \mu \mathrm{l}$ of PBS alone served as control. The remaining two test groups (Group 2 and 3) were intraperitoneally administered with $10 \mu \mathrm{g} / 20 \mu 1$ of SIF (Sperm immobilization factor) from $S$. aureus and $10 \mu \mathrm{g} / 20 \mu \mathrm{l}$ of SAF (sperm agglutination factor) from $E$. coli without under any anesthesia. Each factor was administered on day 1, 3 and 7 and then the mice were sacrificed $48 \mathrm{~h}$ post-inoculation on days 3,5 and 9 .

\section{Body weight profile and tissue somatic indices (TSI \%)}

Initial body weight of mice from each group was taken on the 1st day of experiment and final weight was on the last day of the experiment. On day 3, 5 and 9 mice from each group were sacrificed by cervical dislocation and the various reproductive organs (viz. testis, caudal epididymis and vas deferens) and non reproductive organs (spleen, kidney and bladder) were removed aseptically. The organs were grossly examined and weighed. The TSI (percent tissue/ organ weight in relation to body weight) was evaluated. ${ }^{14}$

\section{Assessment of seminal parameters}

Sperm count: Mice from each group were sacrificed by cervical dislocation and were dissected. The vas deferens was pulled out and placed in freshly prepared $500 \mu$ of PBS buffer $(50 \mathrm{mM}, \mathrm{pH} 7.2)$. Gentle teasing was done to enable the spermatozoa to swim out into the buffer in a glass plate. A fixed volume of $10 \mu \mathrm{l}$ of the sample was placed on a glass slide and examined under the light microscope at 400X magnification. Around six to eight fields were scanned and the mean number of spermatozoa in all the fields was multiplied by 106 . The slides were also assessed for the morphology of spermatozoa in each field to evaluate the respective abnormalities. ${ }^{15}$

Sperm motility: Motility of the sperms extracted from the sacrificed mice was determined by the method of Emmens. ${ }^{16}$

Sperm viability: In order to estimate the percentage of viable sperms, an equal volume of mouse spermatozoa was mixed with $0.5 \%$ eosin and examined under the light microscope at 400X magnification.

\section{Histopathological studies}

Histological analyses of the reproductive organs (testis, caudal epididymis and vas deferens) of mice from each group were carried out. The various reproductive organs and nonreproductive organs were harvested, fixed in $10 \%$ formaldehyde for $24 \mathrm{~h}$ and then embedded in paraffin according to standard histological methods. Serial paraffin sections of $4 \mathrm{~mm}$ were stained with hematoxylin eosin and observed at $400 \mathrm{X}$ magnification for any significant changes in reproductive organs.

\section{Results and discussion}

The microbial factors capable of causing detrimental effects on spermatozoa, on one hand can cause infertility in female mice, but on the other hand show no adverse effects on reproductive performance of male mice. This indicates that the sperm impairing factors that have proved their mettle in one sex may or may not behave in a similar manner in their counterparts. A study citing the similar case has been reported by Reddy et al., ${ }^{8}$ in which they have demonstrated that Nisin, an antimicrobial peptide with spermicidal properties, has shown excellent contraceptive efficacy when administered intravaginally in female, but displayed no adverse effects on the reproductive competence of male rats when administered orally. To the best of our knowledge, no other report citing such comparative work has been reported, though several reports regarding the adverse effect of some chemical compounds e.g. lead, busulfan, triptolide etc. on seminal parameters do exist. In this regard, previously in our laboratory we have also isolated strains of $S$. aureus and E. coli that have been found to produce microbial factors with sperm immobilizing and sperm agglutinating properties in-vitro, respectively. ${ }^{12,13}$ They were also found to possess excellent contraceptive efficacy in female $\mathrm{BABL} / \mathrm{c}$ mice when administered intravaginally. ${ }^{10,17}$ On the lines of Reddy et al., ${ }^{8}$ we also attempted to extrapolate the effect of these microbial factors in male mice. Hence, the present study was designed to evaluate the effect of both these microbial factors i.e. sperm immobilizing factor (SIF) from S. aureus and sperm agglutinating factor (SAF) from $E$. coli on the reproductive potential of male mice. For this, each factor was intraperitoneally administered on day 1, 3 and 7 and then the mice were sacrificed $48 \mathrm{~h}$ post inoculation on days 3,5 and 9 . The various parameters which were evaluated included: body weight profile, seminal parameters, tissue somatic indices and histopathological studies.

\section{Isolation and purification of SIF and SAF}

Both the factors were isolated and purified by the protocol already standardized in our laboratory. ${ }^{12,13}$

\section{Weight profile}

When the weight profile study of the different group of mice was carried out, the results showed that there was a consistent increase in the body weight in the control group during the course of the experiment. However, a mild decrease in body weight was observed in the case of mice in test groups. This mild decrease might be due to a decline in food consumption since weight change depends on a balance between energy expenditure and food consumption. This is in consistence with study by Qin et al., ${ }^{9}$ wherein intraperitoneal administration of Busulfan in male mice led to a mild decrease in the body weight.

\section{Tissue somatic indices (TSI \%)}

When TSI (\%) of various reproductive (testes, cauda and vas deferens) and non-reproductive (spleen, kidney and bladder) organs was examined, it was found that there were no treatment-related changes in the absolute weights of all the organs, except spleen and vas deferens in case of SIF where a significant increase in weight was observed on all the 3 days. However, right cauda showed marked decrease in TSI on all the 3 days and testes showed a marked decrease in TSI on day 9 only. Further, in the case of SAF treated group, left vas deferens showed a considerable increase in TSI on day 3 while the right cauda epididymis showed a significant reduction on days 5 and 9 (Figure 1). The evaluation revealed that no clear pattern could be obtained, hence, reliance on more sensitive end points such as morphological and functional abnormalities of these organs could be helpful to better delineate the alterations.

\section{Evaluation of seminal parameters}

Some of the major determinants of fertilization potential of spermatozoa are based on their microscopic assessment of sperm 
concentration, motility, viability and morphology. In order to assess the treatment-related changes in seminal parameters, all the mice from each group were sacrificed on day 3, 5 and 9 .

Sperm count: Since, the collective result of all the steps of sperm production is provided by sperm count analysis, hence it is considered as one of the most trusted tests for spermatogenesis. In this regard, when mice treated with SIF were assessed for changes in total sperm count, a significant reduction, with respect to control, was observed on all the days of experiment viz. 53\% (on day 3), 80\% (day 5) and $92 \%$ (on day 9). However, in the case of SAF treated group, day 3 witnessed an insignificant reduction in total sperm count whereas a complete inhibition of spermatogenesis was observed on day 5 and 9 (Figure 2a). The results showed that the factor-induced reduction/ complete suppression in sperm count is indicative of the fact that reproductive function of male mice was compromised. Similar results have been reported by Lue et al. ${ }^{18}$ where marked reduction in total sperm count was observed when triptolide was administered orally to male rats.

Motility: It is a well known fact that the sperm motility has a critical influence on successful fertilization, since the highly motile spermatozoa have a greater chance of fertilization. Hence, the \% motility was evaluated in this study. The results showed that on day 3 , in case of SIF and SAF treated mice, it was observed that there were no significant changes in percent motility of the sperms with respect to control (65\%). Day 5 onwards, SIF rendered all the sperms non-motile (Figure 2b) whereas this parameter could not be assessed in case of SAF treated group because of complete loss of spermatogenesis. The results indicated that SIF and SAF not only deteriorate the quantity, but also the quality of spermatozoa, thereby, proving their mettle in reducing the reproductive vigour of male mice.

Viability: Although the true viability of spermatozoa is ultimately defined by their capacity to move and fertilise an egg, but here the viability of mice spermatozoa was investigated using live/dead sperm viability stains. Here, the viability refers really only to integrity of their membranes. When the spermatozoa were incubated with eosin dye, the dead sperm cells with damaged membranes stained pink whereas the live ones did not. The results revealed that the number of viable spermatozoa in both SIF and SAF treated groups was comparable to control $(67 \%)$ on day 3 . However, in the case of SIF, viability reduced to $43 \%$ and $13 \%$ on day 5 and 9 respectively. Since complete inhibition of spermatogenesis was observed on day 5 and 9 in SAF treated group, hence, viability could not be assessed for the same (Figure 2c), thus further indicating that both these microbial factors have the ability to induce functional deformities in spermatozoa. In support, Ayoka et al., ${ }^{19}$ also reported a significant reduction in the sperm viability in test group given Lead $(\mathrm{Pb})$ orally, as compared to control.

Morphology: When all the spermatozoa were examined for morphological changes upon treatment with SIF /SAF, it was observed that there were no morphological changes in spermatozoa on day 3. However, day 5 onwards, SIF treated mice showed separation of sperm heads from tails, termed as decapitation (Figure 3). Even in the absence of a thorough understanding of the mechanism of abnormality induction, however, it appears that the mere presence of abnormalities is indicative of the interference of SIF in reproductive competence of male mice. On the other hand, no morphological alterations were observed in SAF treated mice. In conclusion, SIF and SAF induced alteration of sperm count, motility, viability and morphology is indicative of the fact that reproductive function was severely compromised.

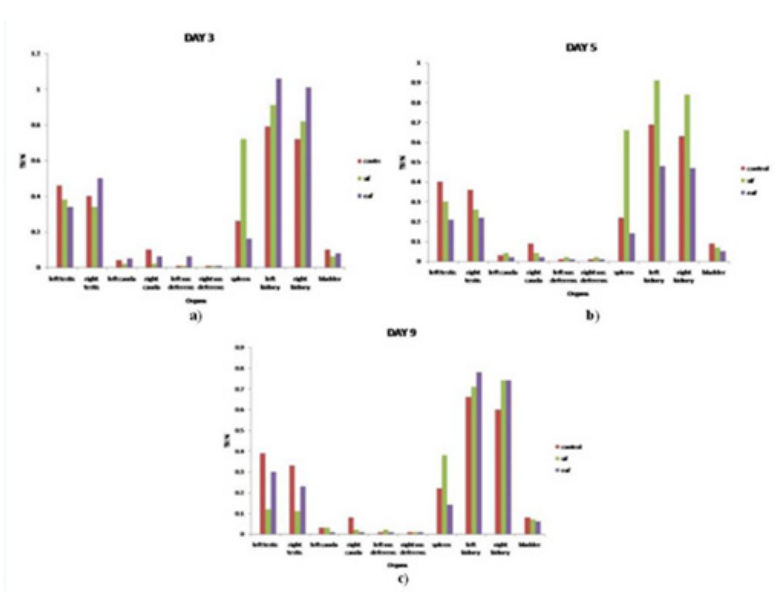

Figure I Tissue somatic indices (\%) of various reproductive and non reproductive organs given SIF/SAF intraperitoneally and sacrificed on day 3 (a), day 5 (b) and day 9 (c).

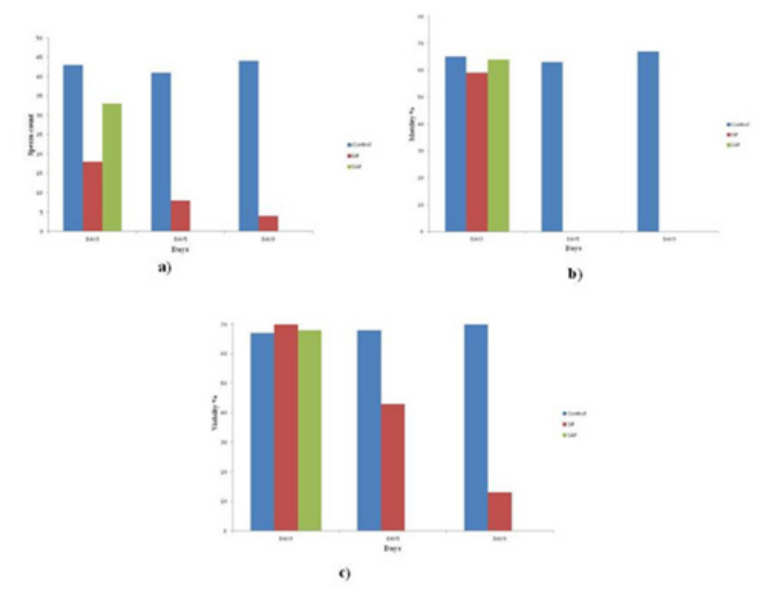

Figure 2 Total sperm count (a), \% motility (b), \% viability (c) of male mice after 3,5 and 9 days of treatment with SIF/SAF.

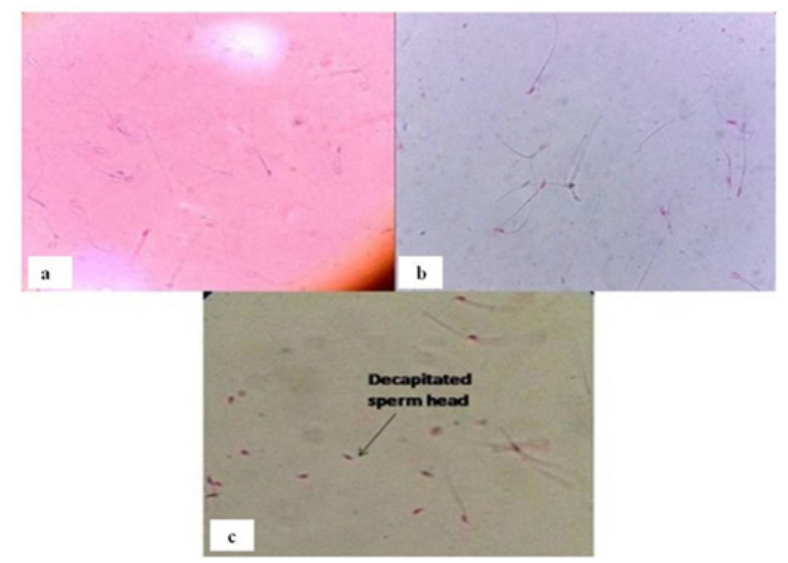

Figure 3 Representative photomicrographs of a) control group showing viable unstained spermatozoa; b) SIF/SAF treated group showing pink stained dead spermatozoa and c) presence of decapitated sperm heads on treatment with SIF.

\section{Histopathological examination}

The control group revealed normal tissue histology in all the reproductive organs. Testis showed regular seminiferous tubule and germinal cell morphology (Figure 4a). Epididymis displayed the well- 
vascularized loose connective tissue present around the epididymal duct (Figure 4b). Further, the normal columnar epithelium was seen in vas deferens (Figure 4c). However, both SIF and SAF treated mice showed that seminiferous tubules in testes have vacant central part with no mature spermatozoa indicating hypospermatogenesis (Figure 4d, 4g). The caudal epididymis showed normal tissue histology (Figure $4 \mathrm{e}, 4 \mathrm{~h}$ ) whereas inflammation was observed in vas deferens (Figure 4f, 4i) in both the groups. Similar histological findings have been reported by $\mathrm{Lu}$ et al., ${ }^{20}$ who observed severe vacuolization of the testicular interstitium when lead $(\mathrm{Pb})$ was administered intraperitoneally in male rats.

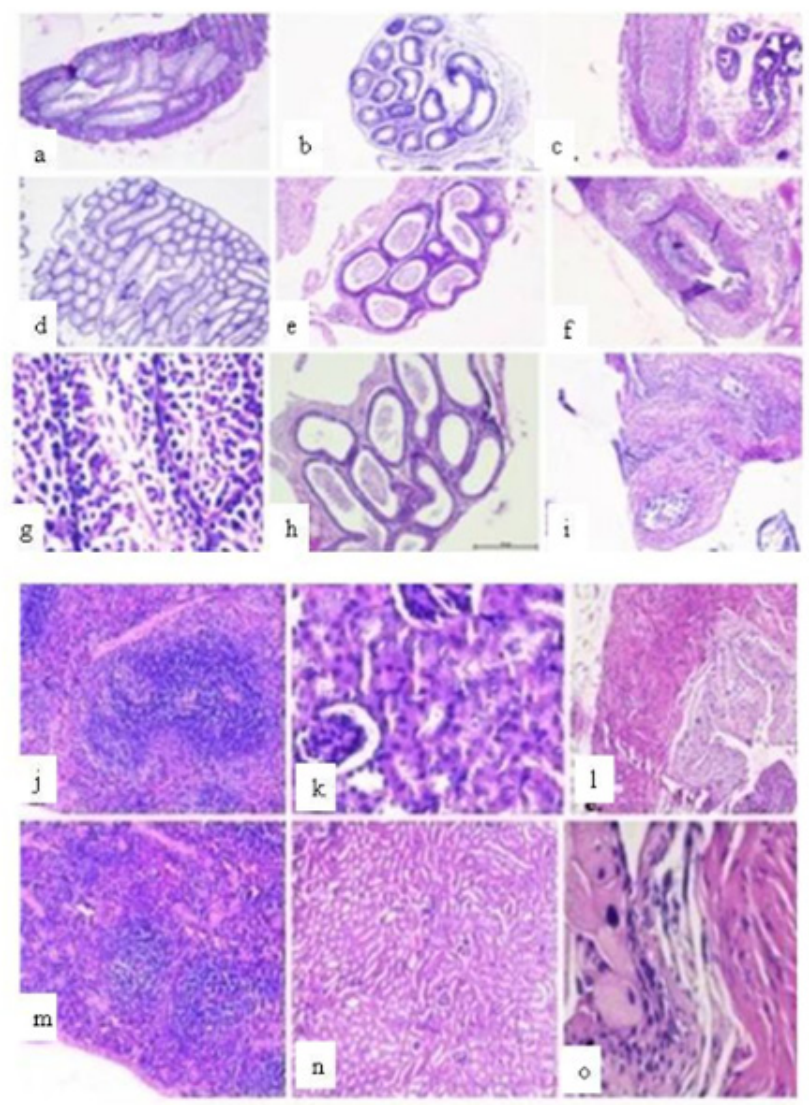

Figure 4 Representative photomicrographs of histopathological examination of reproductive organs viz. testis, cauda epididymis and vas deferens of SIF (df) and SAF (g-i) treated mice compared with the control group receiving PBS $(\mathrm{a}-\mathrm{c})$; non reproductive organs viz. spleen, kidney and bladder of the control group $(\mathrm{j}-\mathrm{l})$ and treated 8groups $(\mathrm{m}-\mathrm{o})$.

However, in striking contrast to this, the tissue histology for nonreproductive organs viz. spleen, kidney and bladder, revealed no abnormalities. All the organs were found to be histologically normal, both in the case of control (Figure $4 \mathrm{j}, 4 \mathrm{k}, 4 \mathrm{l}$ ) as well as treated groups (Figure $4 \mathrm{~m}, 4 \mathrm{n}, 4 \mathrm{o}$ ). Consistent findings were reported by Reddy et al., ${ }^{8}$ wherein they declared that no treatment related alterations in the structural integrity of the reproductive and non-reproductive organs after Nisin treatment were observed compared to their respective controls.

\section{Conclusion}

From the present study, it can be concluded that both SIF and SAF can play an important role in reducing the male reproductive competence.

\section{Conflicts of interest}

The authors declare that there is no conflict of interest.

\section{Acknowledgments}

None.

\section{References}

1. Asif M. A review on spermicidal activities of Azadirachta indica. $J$ Pharmacogn Phytochem. 2013; 5(1):61-79.

2. Connell EB. Barrier contraceptives, spermicides, and periodic abstinence. Curr Opin Obstet Gynecol. 1991;3(4):477-481.

3. DCruz OJ, Uckun FM. Gel-microemulsions as vaginal spermicides and intravaginal drug delivery vehicles. Contraception. 2001;64(2):113-123.

4. DCruz OJ, Yiv SH, Uckun FM. GM-144, a novel lipophilic vaginal contraceptive gel-microemulsion. AAPS Pharm Sci Tech. 2001;2(2): E5.

5. Stephenson J. Widely used spermicide may increase, not decrease, risk of HIV transmission. J Am Med Assoc. 2000;284(8):949.

6. Van Damme L, Chandeying V, Ramjee G, et al. Safety of multiple daily applications of COL-1492, a nonoxynol-9 vaginal gel, among female sex workers. AIDS. 2000;14(1):85-88.

7. Aranha C, Gupta S, Reddy KVR. Contraceptive efficacy of antimicrobial peptide Nisin: in vitro and in vivo studies. Contraception. 2003;69(4):333-338.

8. Reddy KVR, Gupta SM, Aranha CC. Effect of antimicrobial peptide, Nisin, on the reproductive function of rats. ISRN Vet Sci doi. 2011;2011:828736.

9. Qin Y, Liu L, He Y, et al. Testicular busulfan injection in mice to prepare recipients for spermatogonial stem cell transplantation is safe and nontoxic. PLOS One. 2016;11(2):e0148388.

10. Gupta S, Kaur IP, Prabha V. Evaluation of antifertility effect of gel formulation containing Sperm immobilizing factor: in vitro and in vivo studies. Eur J Pharm Sci. 2016;81:67-74.

11. Kala S, Singh A, Singh R, et al. E. coli isolated protein: potential candidates for fertility control in mouse model. Asian J Exp Biol Sci. 2011;2(4):641-647.

12. Prabha V, Gupta $T$, Kaur $\mathrm{S}$, et al. Isolation of a spermatozoal immobilization factor from Staphylococcus aureus filtrates. Can J Microbiol . 2009;55(7):874-878.

13. Kaur K, Prabha V. Sperm impairment by sperm agglutinating factor isolated from Escherichia coli: Receptor Specific Interactions. Biomed Res Int. 2013;548497:1-9.

14. Krishnaiah C, Reddy KP. Dose-dependent effects of fluoride on neurochemical milieu in the hippocampus and neocortex of rat brain. Research report Fluoride. 2007;40(2):101-110.

15. World Health Organization. WHO Laboratory Manual for the Examination and Processing of Human Semen and Sperm. Cervical Mucus Interaction (5 ${ }^{\text {th }}$ edn). 2010;2:1- 286.

16. Emmens CW. The motility and viability of rabbit spermatozoa at different hydrogen ion concentrations. J Physiol . 1947;471-481.

17. Kaur K, Prabha V. Spermagglutinating Escherichia coli and its role in infertility: in vivo study. Micob Pathog. 2014;69-70:33-38.

18. Lue Y, Hikim APS, Wang C, et al. Triptolide: A potential male contraceptive. J Androl. 1998;19(4):479-486.

19. Ayoka AO, Ademoye AK, Oladele AA. Aqueous extract of Allium sativum (Linn.) bulbs ameliorated pituitary-testicular injury and dysfunction in wistar rats with $\mathrm{Pb}$-induced reproductive disturbances. Open access Maced J Med Sci. 2016;4(2):2200-2212.

20. Lu W, Pengcheng X, Yang Z, et al. Effects of lead exposure on sperm concentrations and testes weight in male rats: A meta-regression analysis. J Toxicol Environ Health. 2008;71(7):454-463. 